\title{
Effects of Modulation Defects on Hadamard Transform Time-of-flight Mass Spectrometry (HT-TOFMS)
}

\author{
Joel R. Kimmel, ${ }^{*}$ Facundo M. Fernández, ${ }^{*+}$ and Richard N. Zare \\ Department of Chemistry, Stanford University, Stanford, California, USA
}

\begin{abstract}
In any Hadamard multiplexing technique, discrepancies between the intended and the applied encoding sequences may reduce the intensity of real spectral features and create discrete, artificial signals. In our implementation of Hadamard transform time-of-flight mass spectrometry (HT-TOFMS), the encoding sequence is applied to the ion beam by means of an interleaved comb of wires (Bradbury-Nielson gate), which shutters the ion beam on and off. By isolating and exaggerating individual skewing effects in simulating the HT-TOFMS process, we determined the nature of errors that arise from various defects. In particular, we find that the most damaging defects are: mismatched voltages between the wire sets and the acceleration voltage of the instrument, which cause positive and negative peaks throughout mass spectra; insufficient deflection voltage, which reduces the intensity of real peaks and causes negative peaks that are spread across the entire mass range; and voltage errors as the wire sets return from their deflection voltage to their transmission value, which yield significant reductions in peak intensities, create artificial peaks throughout mass spectra, and broaden real peaks by causing positive peaks to grow in the bins adjacent to them. Because the magnitude of the modulation defects grows as the applied modulation voltage is increased, BradburyNielson gates with finer wire spacing, and hence stronger effective fields for a given applied voltage, were produced and installed. Operating at 10 to $15 \mathrm{~V}$ where errors in the electronics are essentially absent, the most finely spaced gate $(100 \mu \mathrm{m})$ yielded signal-to-noise ratios that were more than two times higher than those achieved with more widely spaced gates. As an alternative method for minimizing skewing effects, HT-TOFMS data were post processed using an exact knowledge of the modulation defects. Nonbinary matrices that mimic the actual encoding process were built by measuring voltage versus time traces and then translating these traces to transmission versus time. Use of these matrices in the deconvolution step led to marked improvements in spectral resolution but require full knowledge of the encoding defects. (J Am Soc Mass Spectrom 2003, 14, 278-286) (c) 2003 American Society for Mass Spectrometry
\end{abstract}

$\mathrm{O}$ ver the past few years, our group has published a series of articles describing Hadamard transform time-of-flight mass spectrometry (HTTOFMS) [1-5]. Other groups have described the application of Hadamard multiplexing to absorption spectroscopy [6], Raman spectrometry [7], photoacoustic depth profiling [8], and capillary electrophoresis [9-11]. In each case, the motivation has been the inherent improvement in signal-to-noise ratio (SNR) offered by multiplexing the signal. In addition to its high SNR values, HT-TOFMS coupled to continuous ion sources offers a $50 \%$ duty cycle over any mass range with the

Published online February 25, 2003

Address reprints requests to: Richard N. Zare, Department of Chemistry, Stanford University, Stanford, CA 94305-5080, USA. E-mail: zare@ stanford.edu

*These authors contributed equally to this work.

'Present address: Department of Chemistry, University of Arizona, Tucson, AZ 85721. potential of reaching $100 \%$ [3]. As a result of its high SNR and duty cycle, the spectral acquisition rates of HT-TOFMS are superior to conventional instruments.

Spectral errors resulting from modulation defects have been described for Hadamard instruments that encode optical signals using machined masks [12] and stationary liquid crystals $[7,8]$. These authors have also developed mathematical correction schemes to compensate for the effects of systematic errors. Hanley [13] recently published a review describing the source and manifestation of these errors in various optical instruments. In the same spirit, we present an analysis of the errors in HT-TOFMS arising from imperfections in the modulation scheme.

HT-TOFMS differs from many other HT methods in that it seeks to encode an ion beam rather than a photon beam. The extended mass ranges of modern TOF mass spectrometers make it impractical to use mechanical chopping when applying Hadamard multiplexing 
schemes to TOFMS. Such encoding would demand the construction of choppers with a large number of slits operated at very high rotational speeds. In our implementation of HT-TOFMS the encoding sequence is applied to the ion beam by means of a BradburyNielson gate (BNG) [14-16], which shutters the ion beam on and off.

Detailed descriptions of how encoding sequences are applied in HT-TOFMS have been presented previously [2], and only a brief review is given here. Ions are accelerated toward a BNG. This ion gate consists of two isolated sets of wires, which are interleaved and lie in the same plane. A pseudorandom sequence of 1's and 0 's, reflecting the first row of a simplex matrix, is generated by a system of shift registers, split into two inverse phases, and used to drive a push-pull amplifier to form a train of square pulses. These two amplified phases are simultaneously applied to the wire sets of the BNG. The wires of the BNG float at the acceleration voltage of the mass spectrometer. A " 1 " in the sequence holds both wire sets at the same voltage (100\% transmission), which is that of the acceleration voltage. $\mathrm{A}$ " 0 " in the sequence raises one wire set above the acceleration voltage and lowers the other wire set below the acceleration voltage by the same amount. Ions incident on the BNG are attracted by one wire set and deflected by the other, causing the ions to diverge from their initial flight path and miss the detector $(0 \%$ transmission). The sequence is applied to the BNG at a rate of 10 $\mathrm{MHz}$. The rapid on/off modulation of the ion beam creates ion packets of assorted sizes. These ion packets interpenetrate one another as they drift through the flight tube toward the detector. Typically, more than one thousand packets exist in the flight tube at the same time. The detected signal is a convolution of the timeof-flight spectra corresponding to these different packets.

Using knowledge of the applied sequence, the signal is deconvoluted to yield the time-of-flight spectrum. The matrix used for deconvolution assumes that the applied pulse shapes are perfectly square and that only two deflection modes exist: $100 \%$ transmission and $0 \%$ transmission. The fidelity of the deconvolution thus depends on the profile of the applied pulses and the discreteness of the sequence as felt by the ions passing through the BNG. This first factor depends on the accuracy of the RF circuitry used whereas the second factor corresponds to the size and strength of the BNG's effective field.

In previous work $[1,2]$ we have noted the existence of spurious peaks in a HT-TOF mass spectrum. Figure 1 presents an example of this behavior; it shows a HTTOF mass spectrum of the salt tetrabutylammonium (TBA) acetate in a water/methanol buffer solution. In addition to the analyte peak in bin 790, the spectrum contains positive and negative peaks with significant intensity in acquisition bins throughout the entire mass range, including those bins immediately adjacent to the real peak. The nature of these unwanted peaks depends

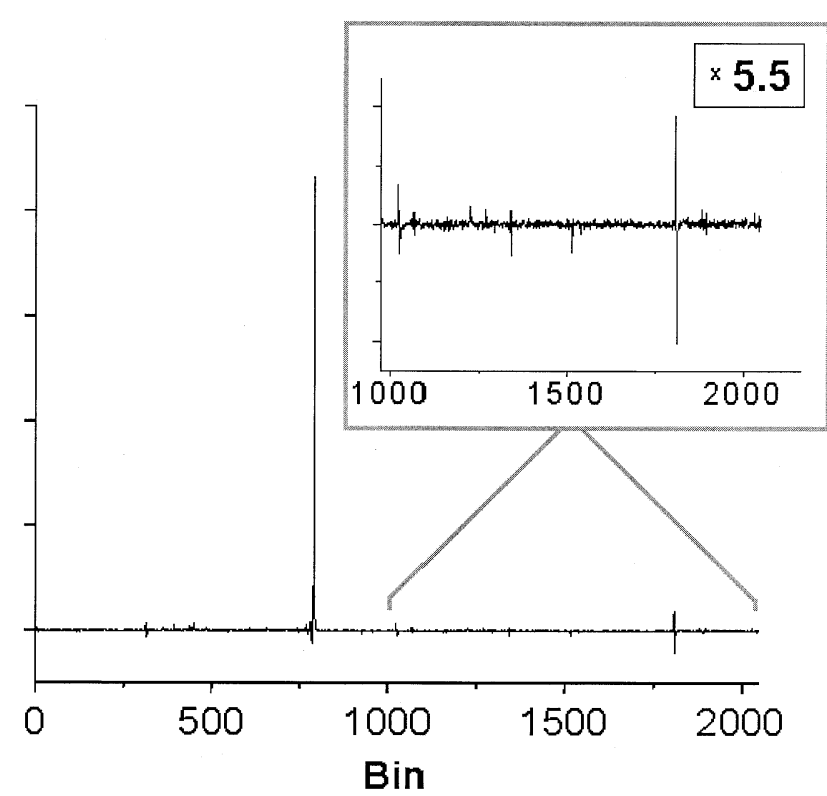

Figure 1. HT-TOF mass spectrum of the salt tetrabutylammonium (TBA) acetate in a water/methanol buffer solution. Discrete spectral echoes caused by modulation errors are evident throughout the entire mass range.

on the composition of the sample, the instrument's operating voltages, and the encoding sequences used. We rule out source fluctuations as the origin of these features because of the rapid modulation rate we use. Based on published work involving the use of the Hadamard transform, we have assumed that these "echoes" are a result of errors in the modulation of the beam, but the exact source of these errors was not previously understood.

To identify the modulation errors in this electronic encoding system, the actual shapes of the voltage pulses applied to the BNGs were determined and used to model the HT-TOFMS process. By isolating and exaggerating individual skewing effects in the model, it has been found that mismatched voltages between the wire sets and the acceleration voltage of the instrument, insufficient deflection voltage, and voltage errors as the wire sets return from their deflection voltage to their transmission voltage, reduce the intensity of analyte peaks and simultaneously produce false peaks in HTTOFMS spectra. Most detrimental are echoes that appear in the acquisition bins on either side of an analyte peak. While all echoes limit the sensitivity of the technique and complicate the spectra, these particular echoes reduce the mass resolution.

It was found that the magnitude of the modulation defects and the intensity of the echoes grew as the applied modulation voltage was increased. In an attempt to reduce the operation voltage of the instrument, BNGs with finer wire spacing, and hence stronger effective fields, were produced and installed. The most promising spectra were obtained using a BNG with a wire spacing of $100 \mu \mathrm{m}$ at deflection voltages between 10 and $15 \mathrm{~V}$. Within our instrument, where ions must be 
deflected off the axis of the flight path by at least 0.07 degrees in order to miss the detector, these spectra had a signal-to-noise ratio more than 2 times greater than that achieved with more widely spaced BNGs at deflection voltages up to $50 \mathrm{~V}$. Echo intensities in the spectra obtained with this $100-\mu \mathrm{m}$ BNG were among the lowest observed.

$\mathrm{A} \mathrm{TBA}^{+}$spectrum obtained with the 100- $\mu \mathrm{m}$ BNG operating at a deflection voltage of $25 \mathrm{~V}$ was used to demonstrate how, with an exact knowledge of the modulation errors, software post-processing can further reduce echo intensity. The processing eliminated the spectral echoes in the bins immediately adjacent to the $\mathrm{TBA}^{+}$peak, narrowing the width of the $\mathrm{TBA}^{+}$peak by $30 \%$ and resolving isotopes that were present.

\section{Experimental}

\section{Reagents}

The sample for all HT-TOFMS experiments was a 200 $\mu \mathrm{M}$ solution of tetrabutylammonium acetate (TBA, Sigma Chemical, St. Louis, MO), $\mathrm{Mw}=301.5 \mathrm{~g} \mathrm{~mol}^{-1}$, in a 50:50 v/v mixture of high purity water $(18 \mathrm{M} \Omega$ $\mathrm{cm}^{-1}$ ) and methyl alcohol (Aldrich Chemical, Milwaukee, WI) with $0.001 \mathrm{M}$ acetic acid (Aldrich) added. Sample solutions were sonicated and filtered with 0.45 $\mu \mathrm{m}$ Puradisc AS disposable cartridges (Whatman, Maidstone, UK) before analysis.

\section{Electrospray Ionization (ESI) HT- TOFMS}

The basic configuration of the ESI HT-TOFMS, including the pseudorandom sequencer generator, the ion optics scheme, the data acquisition system, and the electrospray ionization source have been described previously [4]. The $\mathrm{TBA}^{+}$solution was continuously infused through a $30 \mathrm{~cm}$ long, $100 \mu \mathrm{m}$ i.d. x $360 \mu \mathrm{m}$ o.d. fused-silica capillary (Polymicro Technologies, Tucson, AZ). One end of the capillary was converted to a gold-coated sheathless electrospray emitter following the procedure described by Barnidge et al. [17, 18]. Solutions were nebulized by applying $35 \mathrm{hPa}(0.5 \mathrm{psi})$ to a sealed sample vial connected to the ESI emitter, which was held at $2.5 \mathrm{kV}$. The emitter tip was mounted in a xyz micro-positioning stage opposite the grounded interface of the mass spectrometer $\left(125^{\circ} \mathrm{C}, 2.7\right.$ Torr $)$. A $40 \mathrm{~mm}$ diameter multichannel plate (MCP) detects the ions. The detector is preceded by a 6-mm masking slit that blocks ions deflected by the BNG. The flight distance between the BNG and this mask is approximately $2 \mathrm{~m}$. The MCP signal was preamplified (VT120C, EG\&G Ortec, Oak Ridge, TN) and fed to the discriminator input of a multichannel scaler (TurboMCS, EG\&G Ortec) which was furnished with a 50$\mathrm{MHz}$ clock output for synchronization of the detection and encoding circuits.

The encoding sequence, which is generated by an 11-bit shift registry and applied to the BNG in the form of binary and periodic square voltage pulses, repeats after 2047 100-ns elements have been applied to the wires. When measuring mass spectra, the multichannel scaler summed counts in 2047 bins, with a dwell time of 100 ns. Data were collected on a PC using Turbo MCS software (EG\&G Ortec). Each set of 2047 elements constitutes one scan; 200,000 successive scans were summed to increase spectral intensity. Using software written in $\mathrm{C}++$, data sets were deconvoluted with the inverse Hadamard transform to recover the TOF spectrum.

When optimizing the instrument and when measuring ion deflection efficiency of the BNGs, ion counts were summed in 100-ms bins. The voltages of the ion optics were adjusted to maximize total ion counts each time a new BNG was installed. To ensure that the beam was centered on the gate, three beam profiles were recorded according to our previously described procedure [16]. At the start of each run, ESI emitter position was adjusted for intensity and signal stability.

\section{Bradbury-Nielson Gates}

Bradbury-Nielson gates with wire spacing of 100, 150, and $300 \mu \mathrm{m}$ were produced using a weaving method we developed [16]. Wire spacing was controlled using a mechanically etched polymer wire guide (Ultem 1000, General Electric Plastics, Pittsfield, MA). $20 \mu \mathrm{m}$ goldplated tungsten wire (California Fine Wire, Grover Beach, CA) was used for all BNGs. With the exception of the space between grooves on the wire guide, the four BNGs were identical in design. The central circular active area of each BNG, through which the ions pass, had a diameter of $15 \mathrm{~mm}$. The maximum diameter of the beam entering the gate was estimated to be $5 \mathrm{~mm}$.

\section{Determination of Deflection Efficiency}

While infusing the water:methanol:acetic acid mixture, the total intensity of the ion beam was measured by counting ions with all BNG wires held at $-1250 \mathrm{~V}$, the acceleration voltage applied to the instrument's liner; see Figure 2 (beam on mode). If, in addition to the liner voltage, a constant DC voltage is applied to each set of wires so that they differ from the instrument's liner voltage by an equal magnitude and opposite sign, the gate's deflection efficiency can be measured. The magnitude of the difference between the positive wire set and the liner voltage is termed the deflection voltage. Provided that the deflection voltage is large enough, all ions will be deflected from their initial flight trajectory and will miss the detector. Hence, this mode, in which the voltage of Wire Set 1 is positive and the voltage of Wire Set 2 is negative relative to the acceleration voltage of the ions, is called the beam off mode. The threshold voltage necessary to deflect $100 \%$ of the ion beam depends on the dimensions of the mass spectrometer and the BNG wire spacing. At voltages below the threshold a fraction of the ion beam will pass 


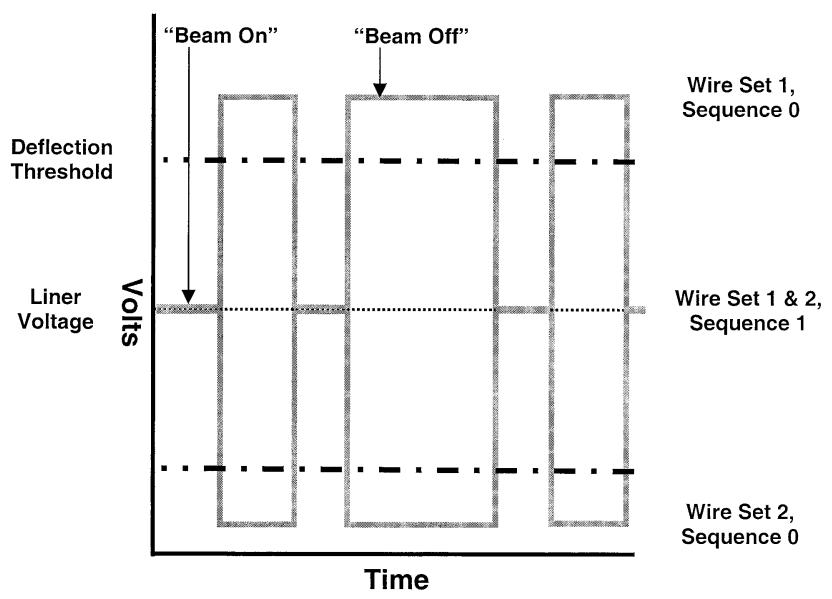

Figure 2. Schematic representation of the electronic modulation in HT-TOFMS. When the voltages of the wire sets equal the acceleration voltage the ion beam passes the gate undeflected. When Wire Set 1 is positive and Wire Set 2 is negative relative to the acceleration voltage ions are deflected. At voltages with magnitudes less than the deflection threshold only a fraction of the beam is deflected.

undeflected. A deflection efficiency curve is obtained by scanning this DC bias voltage between 0 and $50 \mathrm{~V}$ and monitoring the ion counts with 100-ms wide acquisition bins. These curves were fit to a sigmoid function that was used to extract the defect parameters related to incomplete deflection.

\section{Encoding Sequence Pulse Traces}

Traces of the square encoding pulses were obtained by probing one of the wire sets using a $500 \mathrm{MHz}$ digital storage oscilloscope (Waverunner LT 342, LeCroy, Chestnut Ridge, NY). Data were graphically analyzed to determine the rise times (defined as time necessary to reach $90 \%$ of intended voltage), percent overshoot, and settling time (defined as time necessary to settle within $5 \%$ of intended voltage). Figure 3 shows a typical trace.

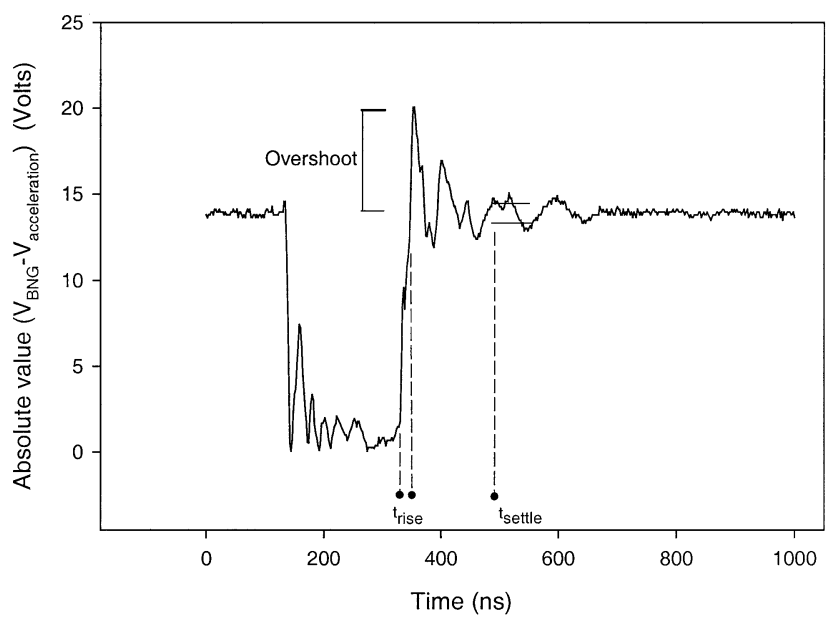

Figure 3. Voltage-time traces for a 100- $\mu \mathrm{m}$ grid, showing defects in rise-time, ringing, and overshoot at a deflection voltage of $15 \mathrm{~V}$.
BNGs were impedance matched to the RF driver using an RC snubber placed on the inner walls of the vacuum chamber housing the ion gate [2]. Optimization of the snubber component values minimized ringing in the sequence, but the ringing could not be completely eliminated. We attribute the residual ringing to power dissipation deficiencies and uncontrolled impedances in our current driver design.

\section{Impulse Response Modeling}

Complete descriptions of the matrices used for encoding the ion beam and of the inverse Hadamard transform used to deconvolute the raw data are found in the work by Harwit et al. [12, 19] and Wilhelmi et al. [20] Crucial to HT-TOFMS, and all other HT techniques, is consistency between the matrices used in the encoding and decoding steps. A data set $x$ is encoded with an $\mathrm{n} \mathrm{x}$ $\mathrm{n}$ simplex matrix, $S_{n}$, yielding a convoluted data set $z$. $S_{\mathrm{n}}^{-1}$, the inverse of the encoding matrix $S_{\mathrm{n}}$, is then applied to $z$ in order to recover the data set $x$. Any discrepancies between the applied encoding scheme and the intended matrix $S_{\mathrm{n}}$ may reduce signal intensities and increase the noise in the mass spectrum. Such discrepancies can arise if either the device delivering the sequence to the system produces errors that skew the sequence or if the effect of a sequence element on the system differs from that which is intended/assumed. The encoding errors that this study focuses on include any experimental factors that distort the elements of the applied $S_{\mathrm{n}}$ matrix in a scan-to-scan invariant manner. In such instances, the deconvoluted spectra contain discrete errors whose intensities, positions, and signs reflect the nature of the encoding defect.

Using the encoding sequence pulse traces described in the previous section, models of the applied pulses were developed in order to simulate the effects of specific modulation defects on HT-TOFMS spectra. The modeling process involves several steps: (1) generation of an impulse vector [13] matching the peak position of $\mathrm{TBA}^{+}$in the experimental spectra, (2) generation of $S_{\mathrm{n}}$ the ideal pseudorandom encoding sequence, (3) calculation of the value of the defective sequence elements at a given modulation voltage (based on the beam deflection profiles), (4) introduction of one or multiple defects in specific portions of the encoding sequence to generate $S_{n}$, the defective encoding sequence, (5) encoding the impulse vector with $S_{n}$, and (6) decoding the convoluted data using the inverse of $S_{\mathrm{n}}$. These operations were performed on a $700 \mathrm{MHz}$ Pentium III-based PC, with 384 MB RAM.

The impulse response vector consisted of a 2047 elements. Element 793 had an arbitrary intensity of 1000 and all other elements had intensities equal to 0 . This vector mimics the HT-TOF mass spectrum of $\mathrm{TBA}^{+}$ obtained in low-resolution mode [5]. No isotopic peaks were added to the simulated spectrum.

The ideal pseudorandom sequence was coded using a series of nested loops that resemble the chained shift 


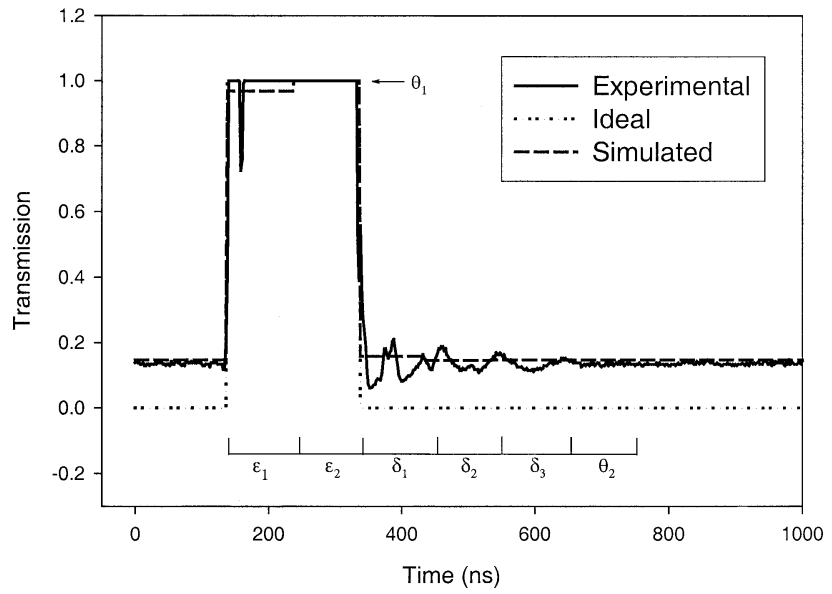

Figure 4. Ideal, experimental, and simulated transmission profiles for a $100-\mu \mathrm{m}$ BNG operated at $15 \mathrm{~V}$. Only the first two defective $\varepsilon$ elements are shown in the plot owing to the duration of the pulse (200 ns)

registers used in the HT-TOFMS electronics. The result is a vector comprised of 1's and 0's. Using simple logical relations, the indices of the following type of sequence elements were found: $\varepsilon_{1}, \varepsilon_{2}, \varepsilon_{3}$ : first, second and third elements after a transmission rising edge (0 to 1$) ; \delta_{1}, \delta_{2}$, $\delta_{3}$ : first, second and third elements after a transmission falling edge ( 1 to 0$) ; \theta_{1}$ : all remaining elements that should be equal to $1 ; \theta_{2}$ : all remaining elements that should be equal to 0 . Figure 4 includes definitions of this nomenclature.

In an ideal case, all $\delta$ and $\theta_{2}$ are 0 while $\varepsilon$ and $\theta_{1}$ are 1. Overshoot and ringing in the applied RF sequence cause the applied voltages to deviate in ways that decrease the transmission of $\varepsilon$-type elements and increase the transmission of $\delta$-type elements. Simultaneously, application of deflection voltages with magnitudes that are less than the deflection threshold yields transmission in the beam off mode, altering the value of all $\delta$ and $\theta_{2}$ type elements.

Within one modulation bin (100 ns) the applied voltage might oscillate several times around the specified value. This ringing-overshoot effect was modeled by fitting the experimentally measured pulse shape to a damped-sine function with variable amplitude depending on the deflection voltage. The generated voltages versus time curves were converted to transmission versus time curves using the experimentally obtained voltage-deflection relationship. The transmission in an acquisition time bin was calculated as the mean value of the transmission in a $100 \mathrm{~ns}$ interval. These transmission values were then used in the construction of skewed modulation sequences consisting of values between 0 and 1.

In some instances, a difference between the magnitude of the applied RF sequence pulses and DC bias voltages continued to induce unwanted deflection in the beam on mode even after the ringing had dampened. This difference in voltage, which was caused by inaccuracies in our home-built power supplies, was used to adjust the value of $\theta_{1}$-type elements. The value of $\theta_{2}$-type elements was simply the transmission efficiency at the applied deflection voltage value.

The skewed sequences were used to generate the simplex matrix by shifting each successive row by one element to obtain $S_{2047^{*}}$. The $S_{2047}^{-1}$ matrix used for deconvolution was computed by inverting the $S_{2047}$ matrix derived from the ideal 2047-element pseudorandom sequence.

\section{Spectral Correction Methods}

Spectral correction methods were demonstrated using an experimental HT-TOF mass spectrum of $\mathrm{TBA}^{+}$. The inverses of the skewed matrices described in the previous section, $\left(S_{2047^{*}}\right)^{-1}$, were calculated and used to deconvolute the experimental data. To efficiently improve the $\mathrm{TBA}^{+}$peak shape the defect parameters used in the correction method were chosen by setting a search grid 0.15 transmission units wide around each of the modeled defect values. The best correction parameters were chosen by visual inspection of the deconvoluted spectrum.

\section{Results}

\section{Ideal, Experimental, and Modeled Pulses}

Similar to when poorly-cut mechanical slits are used to apply encoding sequences in HT optical spectroscopy, skewing of voltage pulses caused by scan-to-scan invariant effects produces echoes in HT-TOF mass spectra. In the encoding scheme described, we expect four potentially detrimental effects: slow rise times, voltage overshoot on the edges followed by ringing, mismatched baselines between the two wire sets, and incomplete deflection in the beam off mode.

Using the methods described earlier, voltage-time traces, such as that displayed in Figure 3, were used to develop transmission-time traces. Figure 4 shows transmission versus time for experimental and ideal $15 \mathrm{~V}$ pulses using a $100 \mu \mathrm{m}$ BNG together with the simulated transmission vector integrated over the discrete time bins.

The downward transmission spike following the rising voltage edge in Figure 4 reflects overshoot as the wire sets move from the deflection voltage to the liner voltage. In voltage versus time plots the relative magnitude of this overshoot was $\sim 55 \%$ (see Table 1 ) and varied little between deflection voltages and BNG wire spacing. The magnitude of this spike in the transmission plot depends on the deflection efficiency of the BNG in the voltage range characteristic of the voltage overshoot. Beyond the edge, the transmission value remains slightly below its maximum for another $40-50$ ns. This unintentional deflection reflects ringing in the $\mathrm{RF}$ voltage that causes the wire sets to oscillate around the liner voltage. In order for overshoot or ringing of 
Table 1. Experimentally-determined modulation pulse characteristics for a $100-\mu \mathrm{m}$ BNG operating at $10 \mathrm{MHz}$.

\begin{tabular}{lccc}
\hline $\begin{array}{l}\text { Deflection } \\
\text { Voltage (V) }\end{array}$ & $\begin{array}{c}\text { Rise Time } \\
\text { (ns) }\end{array}$ & Overshoot & $\begin{array}{c}\text { Settling Time } \\
\text { (ns) }\end{array}$ \\
\hline \hline 5 & 6 & $63 \%$ & 85 \\
15 & 5 & $61 \%$ & 84 \\
25 & 5 & $55 \%$ & 84 \\
40 & 6 & $48 \%$ & 84 \\
\hline
\end{tabular}

Rise times were recorded when the pulses reached $90 \%$ of the desired modulation voltage. Percent overshoot was calculated by comparing the maximum to the set value. Settling time was defined as the time necessary for the voltage to dampen within $5 \%$ of the set value.

this sort to disturb the intended trajectory of the ion beam, the ion gate must demonstrate significant deflection at voltages near the settling value. As the magnitude of the ringing decreases, the transmission approaches a constant, maximum value. Differences between the final value of the wires and the liner voltage of the instrument may prevent this maximum from equaling 1 . The transmission remains at this stabilized value until the next sequence 0 .

All that is necessary to deflect the beam completely, as a sequence 0 implies, is the application of a voltage with magnitude greater than the deflection threshold. Thus, overshoot on the transition from 1 to 0 cannot harm the applied sequence. In fact, if the magnitude of the intended voltage is below the deflection threshold, overshoot in this direction will momentarily improve performance. In Figure 4, the transmission falls rapidly towards $0 \%$ as the voltage overshoots the intended value. The voltage then recovers, rings sinusoidally, and finally settles at the applied deflection voltage. In Figure 4, the $15 \mathrm{~V}$ that are applied are not sufficient to induce complete deflection. As a result, the transmission oscillates around and settles at a value near 0.15 . Rise and fall times were measured to be on the order of $5 \mathrm{~ns}$ for all wire spacing and modulation voltages. While slow transitions could significantly alter the sequence, 5 ns seemed to be negligible in these experiments using 100 ns modulation elements.

The simulated pulses compensate for each of these effects by adjusting the values of the 100 ns elements equal to the mean value of the real data during the same time span. The simulated pulse displayed in Figure 4 takes into consideration all of the factors discussed above. Sequence elements past the points where the ringing has settled were modeled using the parameters $\theta_{1}$ and $\theta_{2}$ for the beam on and beam off modes, respectively (1.000 and 0.146 in the example shown in Figure 4). For the $15 \mathrm{~V}$ trace displayed in Figure 4, the values of the edge defect parameters were $\varepsilon_{1}=0.968$ and $\varepsilon_{2}=1.000$ for the 0 to 1 transition, and $\delta_{1}=0.157$, $\delta_{2}=0.146$, and $\delta_{3}=0.140$ for the 1 to 0 transition. In order to investigate how masking defects vary with experimental parameters, similar plots were derived from voltage versus time data for deflection voltages between 0 and $40 \mathrm{~V}$. As described later, the skewed $S$ matrices, $S_{2047}{ }^{*}$, built from these results were used to simulate HT-TOF mass spectra of $\mathrm{TBA}^{+}$and to correct the experimental spectra.

\section{Impulse Response Method Calculations}

As a first step toward understanding the manifestation of modulation errors in the HT-TOF mass spectra, each type of electronic sequence skewing was simulated using the impulse-response method. Four separate skewed S matrices, $S_{2047^{*}}$, with each of the defect parameters artificially accentuated, were applied to the impulse response vector and deconvoluted with the inverse of the ideal matrix, $\left(S_{2047}\right)^{-1}$, to determine specific effects on spectra. Figure 5 displays the results for (a) $\theta_{1}, \varepsilon_{1}, \varepsilon_{2}$, and $\varepsilon_{3}$ equal to 0.9 , (b) $\theta_{2}, \varepsilon_{1}, \varepsilon_{2}$, and $\varepsilon_{3}$ equal to 0.3 (c) $\varepsilon_{1}$ equal to 0.9 and (d) $\varepsilon_{1}$ equal to 0.3 . Conceptually, these conditions mimic (a) differences between the wire voltages and the instrument's liner voltage in the beam on mode (b) voltages below the deflection threshold in the beam off mode (c) rise times, overshoot, and/or ringing on the rising edge of a transmission pulse, and (d) rise time and/or ringing on the falling edge of a transmission pulse. The baseline near the simulated $\mathrm{TBA}^{+}$peaks in Figure 5 has been magnified to investigate changes in spectral resolution. With the exception of case (d), all spectra contain obvious echoes. While the echoes are easily identifiable in this simple spectrum of a known compound, interpretation can become complicated when spectra contain multiple unknown peaks, each producing their own echoes. Most notable and destructive are the echoes in the bins on each side of the real peak, which effectively lessen the resolution of spectrum. These echoes are most prominent in the spectra suffering from errors on the 0 to 1 transition edge (Case c). Another intense echo group is that centered in bin 1812, which appears in cases $a, b$, and $c$. All echoes resulting from operating at a deflection voltage below the deflection threshold (Case b) have negative intensity. Also noteworthy in Figure 5 is the decrease in signal that accompanies the appearance of echoes. The simulated peak has an arbitrary intensity of 1000 units. In Cases b and c, where the echoes have their greatest intensity, peaks heights have reduced to 930 and 810 , respectively.

\section{Modeling Real Spectra Using Measured Defect Parameters}

Matrices derived from the pulse traces were used to predict HT-TOFMS spectra at voltages between 5 and $40 \mathrm{~V}$ using a $100-\mu \mathrm{m}$ BNG (Figure 6a). There are several trends apparent in these spectra that correlate well with the experimental data (Figure 6b). Signal intensity increases toward a maximum as voltage in increased, while echo intensity is minimal below $15 \mathrm{~V}$. These observations reflect the fact that, because deflection efficiency is poor below the threshold deflection voltage 

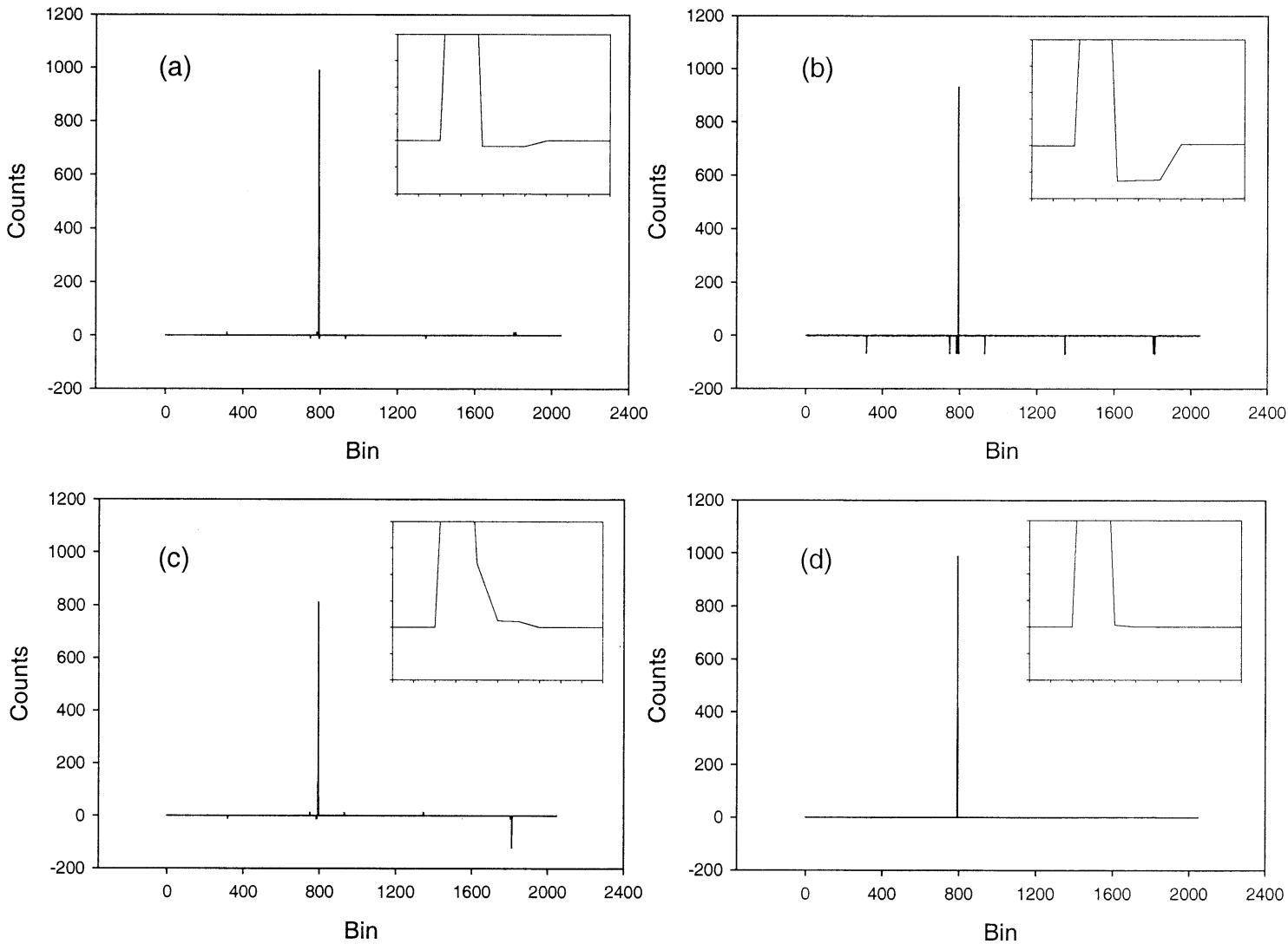

Figure 5. Simulated HT-TOFMS spectra of TBA ${ }^{+}$limiting modulation errors to only a single type in each case: (a) $\theta_{1}, \varepsilon_{1}, \varepsilon_{2}$, and $\varepsilon_{3}$ equal to 0.9 ; (b) $\theta_{2}, \delta_{1}, \delta_{2}$, and $\delta_{3}$ equal to 0.3 ; (c) $\varepsilon_{1}$ equal to 0.9 ; and (d) $\delta_{1}$ equal to 0.3 .

(25 $\mathrm{V}$ in this case), the amplitude of ringing and the magnitude of overshoot are not large enough to significantly distort the modulation sequence in this voltage

(a)

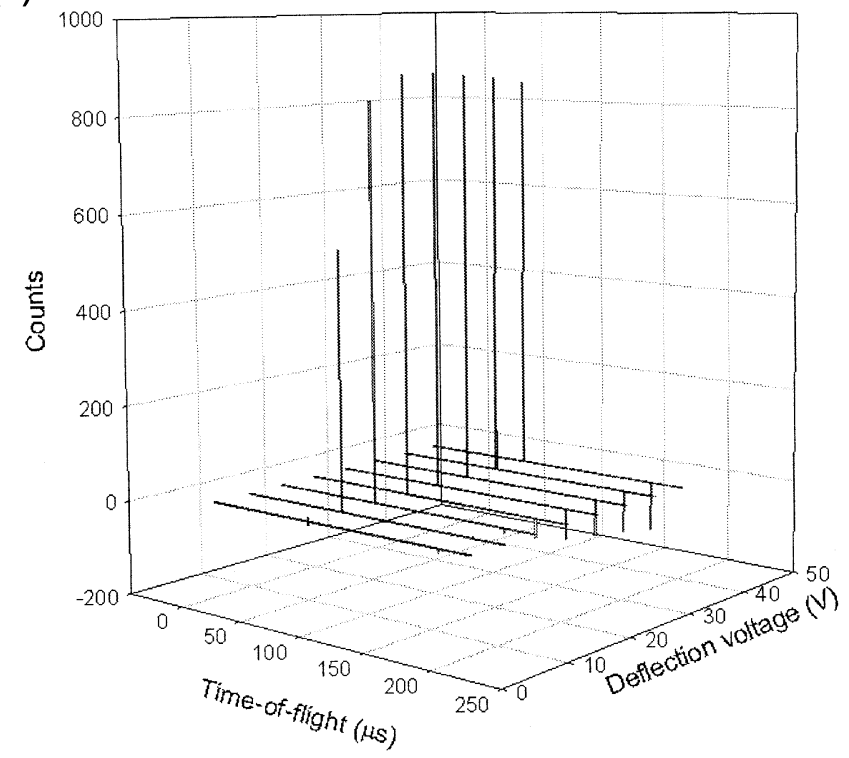

range. At voltages above the threshold, where deflection can no longer improve, the magnitudes of overshoot, ringing, and mismatching are large enough to skew

(b)

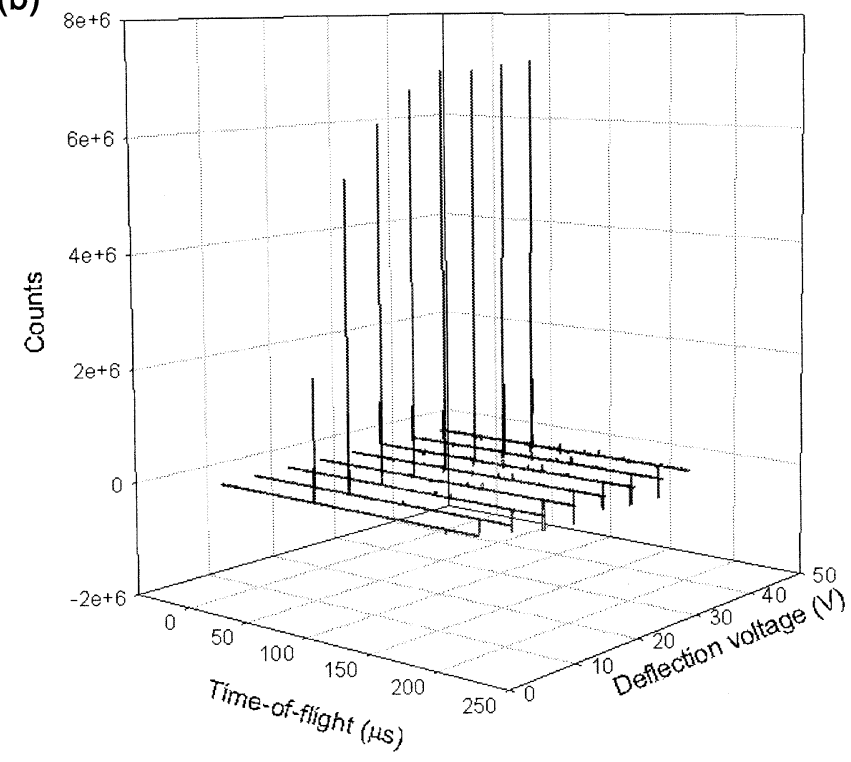

Figure 6. (a) Simulated and (b) experimental HT-TOFMS spectra of $\mathrm{TBA}^{+}$between deflection voltages of 5 and 40 volts using a 100- $\mu$ m BNG. 

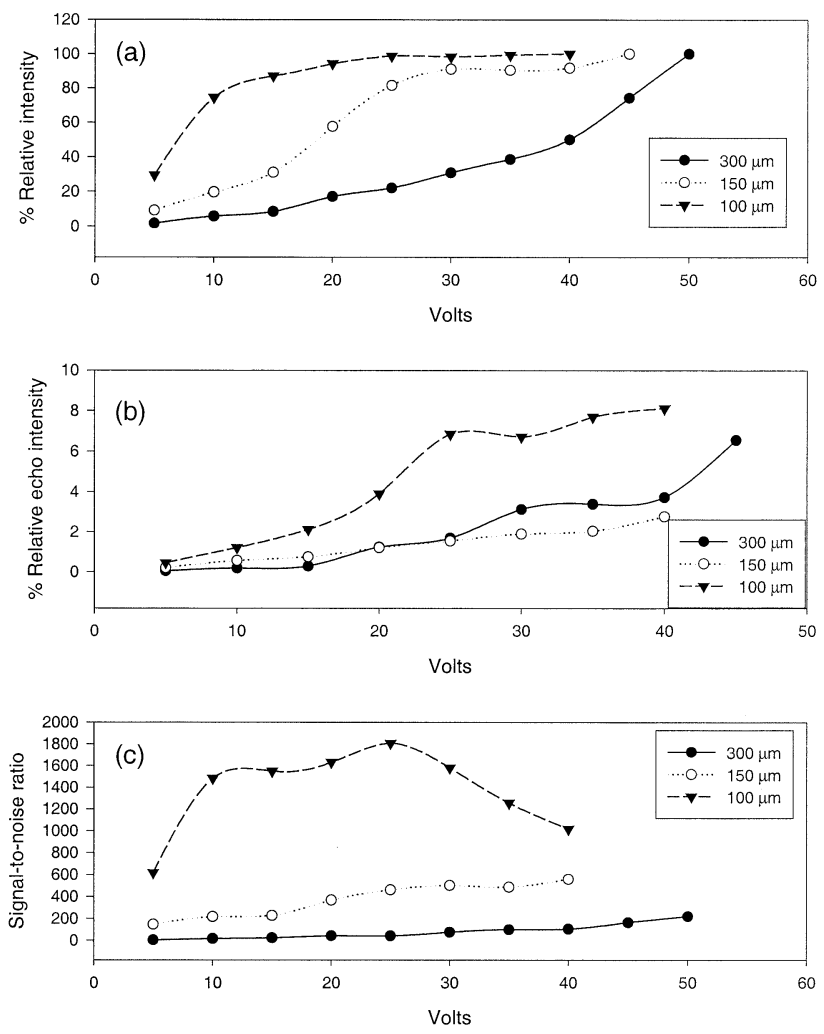

Figure 7. (a) Intensity of $\mathrm{TBA}^{+}$peak in HT-TOFMS spectra; (b) intensity of echo in bin 1812; and (c) signal-to-noise ratio in $\mathrm{TBA}^{+}$ spectra, calculated as the $\mathrm{TBA}^{+}$peak intensity divided by the intensity of the random noise ( $3 \sigma$ of echo-free region of baseline).

significantly the intended deflection of the beam, and their effects become more pronounced in the spectra. Signal intensities begin to fall off, and echo intensities grow rapidly. These trends suggest that the operational deflection voltage must be lowered to improve performance.

\section{Effect of BNG Wire Spacing on Deflection Efficiency and $S N R$}

Optimizing the modulation voltage turns out to be a balance between skewing effects; the effects of ringing and overshooting are minimized as the modulation voltage is lowered, but low voltages (below the deflection threshold) lead to decreased signal intensities. Hence, for best results, the instrument must operate at the deflection threshold. In an effort to reduce the voltage of the deflection threshold to a point where the impact of overshoot and ringing is insignificant, BNGs with finer wire spacing, and consequently stronger effective field strengths, were made and installed.

HT-TOF mass spectra of $\mathrm{TBA}^{+}$were acquired using BNGs with wire spacings of 300,150 , and $100 \mu \mathrm{m}$ at deflection voltages between 0 and $50 \mathrm{~V}$. The $\mathrm{TBA}^{+}$peak intensity, the intensity of the echo peak in bin 1812 relative to the $\mathrm{TBA}^{+}$peak intensity, and the signal-tonoise ratio (SNR) were recorded for each of these spectra. These results are plotted in Figure 7.
Figure 7a compares the relative peak intensities acquired with the three BNGs, each normalized to their own maximum. In each case, we see peak intensity growing as voltage is increased, reflecting an improvement in deflection efficiency. The 300- $\mu \mathrm{m}$ BNG continues to improve across the entire voltage range, suggesting that it never reaches $100 \%$ deflection of the beam. The $150 \mu \mathrm{m}$ BNG rises quickly at $20 \mathrm{~V}$, and levels off between 30 and 45 volts. As expected, the 100- $\mu$ m BNG displayed the most promising results. It rises quickly, reaching $75 \%$ of its maximum intensity at $10 \mathrm{~V}$ and reaching its maximum intensity after $20 \mathrm{~V}$.

Figure $7 \mathrm{~b}$ plots the relative intensity of the echo peak in bin 1812 compared to the $\mathrm{TBA}^{+}$peak. At low voltages the three BNGs display similar values. Near 15 $\mathrm{V}$, the intensity of the echoes observed when using the $100 \mu \mathrm{m}$ BNG begins to rise rapidly. This fact demonstrates the strong control the 100- $\mu \mathrm{m}$ BNG has on the trajectory of the beam. The magnitude of the ringing, overshoot, and mismatching is no greater with this BNG than with any others, but these small fluctuations in the applied voltage significantly distort the profile of modulated beam. Thus, an increase voltage gives a larger signal, as shown in Figure $7 \mathrm{a}$, but also gives a larger echo, as shown in Figure $7 \mathrm{~b}$. Beyond a certain voltage, the echo peak grows faster than the signal for a given BNG spacing.

Figure 7c compares the intensity of the $\mathrm{TBA}^{+}$peak to regions of the spectrum containing only random noise. Modulating a $\mathrm{TBA}^{+}$sample at $15 \mathrm{~V}$, the $100-\mu \mathrm{m}$ BNG demonstrated a SNR of nearly 1500 with echo intensities that are $\sim 2 \%$ of the $\mathrm{TBA}^{+}$peak intensity. This SNR value is more than two times that of any observed with the more widely spaced gates over the entire $50 \mathrm{~V}$ range. While the decreased wire spacing reduces ion transmission $(83 \%$ for a $100-\mu \mathrm{m}$ BNG versus $94 \%$ for a $300-\mu \mathrm{m}$ BNG), the large increase in SNR suggests that the sacrifice is worthwhile. Beyond $30 \mathrm{~V}$ the SNR begins to fall, indicating that mismodulation of the beam at the BNG is becoming significant.

\section{Spectral Correction Methods}

A separate, but complementary, approach to reducing echoes caused by distortion that remains invariant, scan to scan, involves post-processing of the acquired data. Figure 8a shows the $\mathrm{TBA}^{+}$peak shape when the experimental data are deconvoluted using $S^{-1}$. If instead of the ideal $S^{-1}$ matrix, the defective $\left(S^{*}\right)^{-1}$ matrix is used in the deconvolution process, echo intensities decrease and peak shapes are improved. In Figure $8 b$, post processing has reduced the $\mathrm{TBA}^{+}$peak width by $\sim 30 \%$, resolving $\mathrm{TBA}^{+}$isotope peaks.

This correction approach would be completely satisfactory if the masking errors were fixed over time. However, our experience with the present modulation electronics suggests that the errors vary too much, run to run, for this approach to be practical. For this reason, 

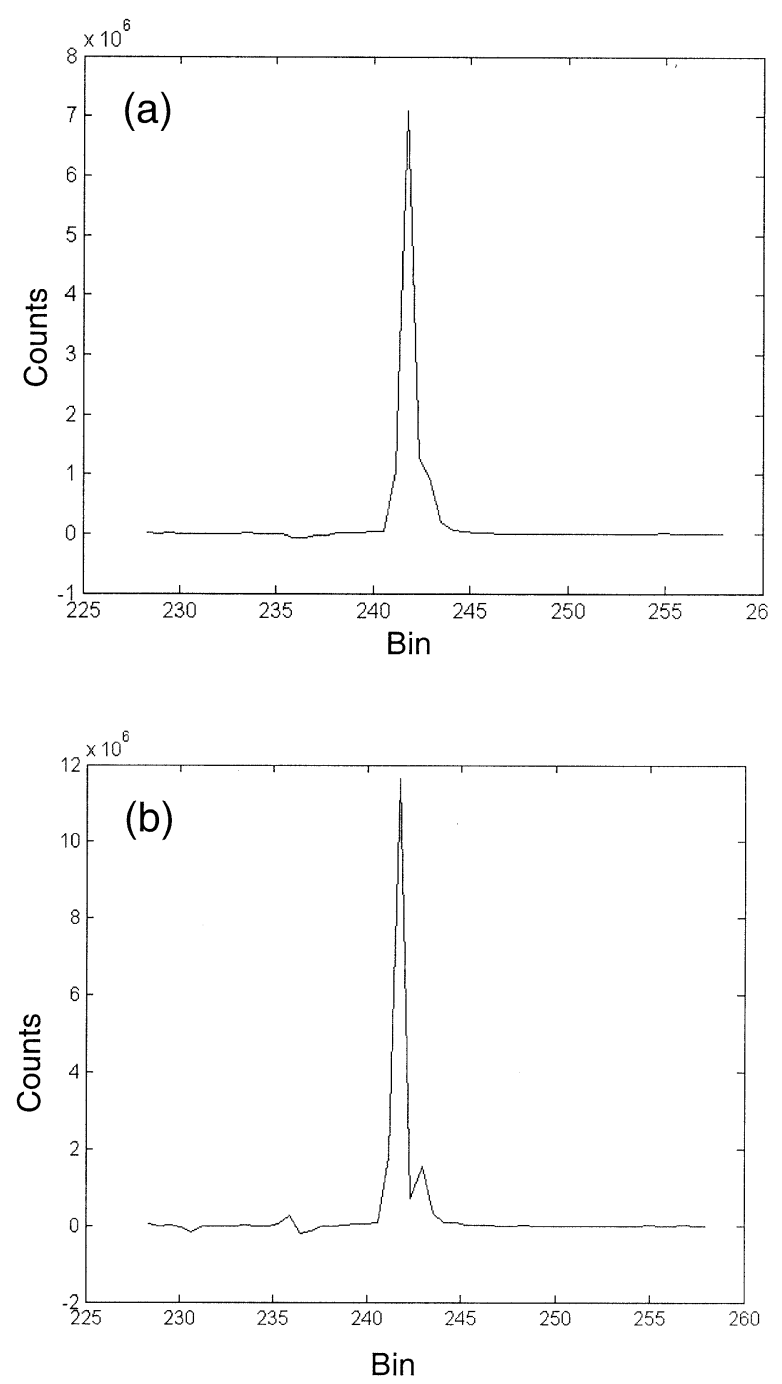

Figure 8. Signal recovery from a $\mathrm{TBA}^{+}$spectrum obtained with a $25 \mathrm{~V}$ modulation voltage and a $100-\mu \mathrm{m}$ grid. (a) Spectrum deconvoluted using the inverse of an ideal simplex matrix and (b) spectrum deconvoluted with the inverse of a simplex matrix generated to include the defective sequence.

efforts are presently being made to re-engineer BNG driver electronics in order to reduce significantly overshoot and ringing. All spectra shown in this study were acquired using modulation bin widths of $100 \mathrm{~ns}$, which limits the achievable mass resolution. This fact explains why Figure 8 does not display the resolution that might be expected for a 2-m flight path. We anticipate that improved modulation electronics will also allow us to reduce this bin width and consequently increase the mass resolution.

\section{Acknowledgments}

JRK was supported by the American Chemical Society, Division of Analytical Chemistry Fellowship, sponsored by Merck \& Co. FMF thanks Fundación Antorchas (Buenos Aires, Argentina) for financial support. This work was supported by the U.S. Air Force Office of Scientific Research.

\section{References}

1. Brock, A.; Rodriguez, N.; Zare, R. N. Anal. Chem. 1998, 70, 3735-3741.

2. Brock, A.; Rodriguez, N.; Zare, R. N. Rev. Sci. Instrumen. 2000, 71, 1306-1318.

3. Zare, R. N.; Fernandez, F. M.; Kimmel, J. R. Hadamard Transform Time-of-flight Mass Spectrometry: More Signal, More of the Time. Angew. Chem. Int. Ed. 2003, 42, 30-35.

4. Fernandez, F. M.; Vadillo, J. M.; Kimmel, J. R.; Wetterhall, M.; Markides, K. E. Rodriquez. N.; Zare, R. N. Anal. Chem. 2002, 74, 1611-1617.

5. Fernandez, F. M.; Vadillo, J. M.; Engelke, F.; Kimmel, J. R.; Zare, R. N.; Rodriquez, N.; Wetterhall, M.; Markides, K. E. J. Am. Soc. Mass Spectrom. 2001, 12, 1302-1311.

6. Tilotta, D. C. Talanta 1990, 37, 61-69.

7. Tilotta, D. C.; Freeman, R. D.; Fateley, W. G. Appl. Spectrosc. 1987, 41, 1280-1287.

8. Fateley, W. G.; Hammaker, R. M.; Paukstelis, J. V.; Wright, S. L.; Orr, E. A.; Mortensen, A. N.; Latas, K. J. Appl. Spectrosc. 1993, 47, 1464-1470.

9. Kaneta, T.; Yamaguchi, Y.; Imasaka, T. Anal. Chem. 1999, 71, $5444-5446$.

10. Kaneta, T. Anal. Chem. 2001, 73, 540A-547A.

11. Kaneta, T.; Kosai, K.; Imasaka, T. Anal. Chem. 2002, 74, 2257-2260.

12. Tai, M. H.; Harwit, M.; Sloane, M. J. A. Appl. Spectrosc. 1975, 14, 2678-2685.

13. Hanley, Q. S. Appl. Spectrosc. 2001, 55, 318-330.

14. Vlasak, P. R.; Beussman, D. J.; Davenport, M. R.; Enke, C. G. Rev. Sci. Instrumen. 1996, 67, 68-72.

15. Stoemer, C. W.; Gilb, S.; Friedrich, J.; Schoos, D.; Loeb, L. B. Rev. Sci. Instrumen. 1998, 69, 1661-1664.

16. Kimmel, J. R.; Engelke, F.; Zare, R. N. Rev. Sci. Instrumen. 2001, 72, 4354-4357.

17. Barnidge, D. R.; Nilsson, S.; Markides, K. E. Anal. Chem. 1999, 71, 4115-4118.

18. Barnidge, D. R.; Nilsson, S.; Markides, K. E.; Rapp, H.; Hjort, K. Rap. Comm. Mass Spectrom. 1999, 13, 994-1002.

19. Harwit, M.; Sloane, M. J. A. Hadamard Transform Optics. Academic Press, Inc.: New York, 1979.

20. Wilhelmi, G.; Gompf, F. Nucl. Instr. Meth. 1970, 81, 36-44. 